\title{
CONTRIBUIÇÃO DAS TEORIAS DE ENFERMAGEM PARA A CONSTRUÇÃO DO CONHECIMENTO DA ÁREA*
}

\author{
Telma Ribeiro Garcia** \\ Maria Miriam Lima da Nóbrega***
}

\section{Resumo}

Neste ensaio são discutidos alguns aspectos acerca do fenômeno conhecimento e da atividade de conhecer, que servem ao propósito de situar a perspectiva a partir da qual as autoras compreendem qual tem sido a contribuição das teorias de enfermagem para a construção do conhecimento da área. As teorias de enfermagem selecionam, definem e interrelacionam conceitos representativos de fenômenos que estão no domínio de interesse da profissão. Garantir a compreensão e o reconhecimento intersubjetivo do significado dos conceitos incluídos nesses referenciais teóricos é um aspecto básico, pois eleva seu potencial de aplicabilidade prática, seja no ensino, na pesquisa ou na assistência de enfermagem. Descritores: conhecimento de enfermagem; teorias de enfermagem; linguagem profissional de enfermagem

\begin{abstract}
In this essay some aspects concerning the phenomenon of knowledge and of the activity of knowing are taken into consideration, serving to the authors' purpose to point out the perspective from which they understand which have been the contribution of the nursing theories for the construction of the knowledge of the area. The nursing theories select, define and interrelate concepts representative of phenomena that are in the nursing domain of interest. To guarantee the understanding and the recognition of the meaning of the concepts included in these theoretical frameworks is a basic aspect, therefore it raises their potential of practical applicability, either in education, research or nursing care.

Descriptors: nursing knowledge; nursing theories; professional nursing language

Title: Contribuction of nursing theories to build knowledge in the area
\end{abstract}

\section{Resumen}

En este ensayo se toman en consideración algunos aspectos referentes al fenómeno del conocimiento y de la actividad de conocer, sirviendo al propósito de las autoras de precisar la perspectiva a partir de la cual entienden cuál ha sido la contribución de las teorías de enfermería para la construcción del conocimiento de enfermería. Las teorías de enfermería seleccionan, definen y correlacionan conceptos que son representativos de fenómenos que están en el dominio de interés de la profesión. Garantizar la comprensión y el reconocimiento del significado de los conceptos incluidos en estos referenciales teóricos es un aspecto básico, desde que aumenta su potencial de aplicabilidad práctica, en la educación, la investigación o en la asistencia de enfermería.

Descriptores: conocimiento de enfermería; teorías de enfermería; lenguaje profesional de enfermería

Titulo: Contribución de las teorías de enfermería para la construcción del conocimiento del área

\section{Introdução}

Em Introdução ao pensar, Alfredo Buzzi(1) afirma que a casa primeira que o ser humano fabrica para morar é a casa do conhecimento, fazendo a ressalva de que, nem sempre, ele é encontrado satisfeito sob o teto construído. Essa satisfação prima por ser variável no tempo porque, embora nasça em um mundo já dotado de uma dada interpretação, ao dialogar consigo próprio, com seus semelhantes e com as coisas que o cercam, o ser humano, ao ir vivendo, procura um ajuste intelectual com o contorno, seja para adaptar-se a ele ou para modificá-lo( ${ }^{(2,3)}$. Nessa atividade, vai provocando inovações, evoluções e/ou revoluções constantes na casa do conhecimento, que não deve ser, portanto, compreendida como definitivamente concluída.

Tomado no sentido mais consensual acerca do termo no seio da comunidade lingüística, conhecimento, do ponto de vista filosófico, significa "a apropriação de algo pelo pensamento, como quer que se conceba essa apropriação: como definição, como percepção clara, apreensão completa, análise, etc."(4:454) Pode-se acrescentar, ainda, que todo conhecimento comporta necessariamente: a) uma competência, ou aptidão para produzir conhecimento; b) uma atividade cognitiva, que se realiza em função da aptidão para produzir conhecimento; c) um saber resultante dessas atividades ${ }^{(5)}$.

Conhecer é uma atividade fundamental para o ser humano, através da qual busca atribuir significado à multiplicidade de fenômenos que o cercam, sejam os referentes a objetos físicos (cadeira, árvore, bicicleta, etc.), a pessoas (vizinho, estudante, padre, mãe, amigo, etc.), a eventos (nascimento, vida, morte, etc.) ou a idéias abstratas (princípios morais, doutrinas filosóficas ou idéias como justiça, exploração ou compaixão, etc.), entre outras possíveis classes de fenômenos que se possam citar.

Conforme usado na afirmativa anterior, o termo fenômeno aplica-se a todos os aspectos da realidade interna e externa, que existem como entidades pré-lingüísticas e que adotam um significado lingüístico a partir da percepção, imaginação, reconhecimento e experiência humanos ${ }^{(6)}$; significado, referese ao conceito, isto é, à "imagem mental que temos dos objetos, eventos, acontecimentos, elementos culturais, etc"(7:32). Por seu turno, atribuir significação corresponde a efetivar a união entre um significante (o rótulo lingüístico com que se "batizou" um dado fenômeno) e um significado (a imagem mental evocada pelo rótulo lingüístico com que se "batizou" o fenômeno). Atribuir significação pressupõe necessariamente a atividade cognitiva, prévia, de identificação e de nomeação do fenômeno; pressupõe, também, a necessidade de definição dos atributos particulares do fenômeno a que se aplicou um determinado rótulo lingüístico. De modo geral, esses atributos, em uma atividade analítica de disjunção, separam e distinguem o fenômeno particular ou, em uma atividade sintética de conjunção, o articulam e associam a outro(s) fenômeno(s).

Como se pode deduzir, a atividade de nomear e de atribuir significado aos fenômenos requer o uso da linguagem, ou seja, o uso de um sistema organizado de signos lingüísticos

\footnotetext{
* Originalmente apresentado no painel "Os referenciais teóricos a nortear a pesquisa em Enfermagem", durante o $11^{\circ}$ Seminário Nacional de Pesquisa em Enfermagem - SENPE, Belém - PA, maio de 2001. **Enfermeira. Doutora em Enfermagem pela EERP-USP. Professora Adjunto IV do Departamento de Enfermagem de Saúde Pública e Psiquiatria, Centro de Ciências da Saúde, Universidade Federal da Paraíba. Pesquisadora CNPq. ***Enfermeira. Doutora em Enfermagem pela UNIFESP/EPM. Professora Adjunto IV do Departamento de Enfermagem de Saúde Pública e Psiquiatria, Centro de Ciências da Saúde, Universidade Federal da Paraíba. Pesquisadora CNPq.

E-mail do autor: tegarcia@ccs.ufpb.br
} 
que, entre outros aspectos, se caracteriza por ser indefinidamente variável no tempo e no espaço, fruto da elaboração do pensamento, adquirido por aprendizagem ${ }^{(7)}$, e utilizado para determinados fins.

A linguagem elaborada pelo ser humano funciona como espelho de sua representação do mundo e do pensamento, como instrumento de comunicação e como forma de ação ou lugar de interação. Como espelho, possibilita ao ser humano representar seu pensamento, seu conhecimento, sua visão de mundo; como instrumento, comunicar-se com os outros, transmitindo-lhes mensagens (ou informações); finalmente, como forma de ação ou lugar de interação, agir, reagir e interagir com os outros membros da sociedade, de modo finalisticamente orientado(8).

Tendo por base a discussão anterior, sintetizamos alguns pontos que consideramos fundamentais para a continuidade deste texto: a) em um sentido abrangente, podese afirmar que conhecer é operar, com a ajuda do pensamento e da linguagem, uma intervenção organizadora no caos aparente do mundo fenomenal que nos cerca; b) a atividade de conhecer é condicionada por fatores físicos, biológicos, psicológicos, pessoais, interpessoais e coletivos, e plasmada pela formação sócio-cultural em cujo contexto vamos vivendo; c) o conhecimento, assim como a linguagem de que nos utilizamos para conhecer, é um fenômeno evolutivo, sujeito a enriquecimentos progressivos e a alterações mais ou menos profundas, destinados (os enriquecimentos e as alterações) às nossas necessidades de ajuste intelectual com o meio ambiente, em um dado espaço e tempo.

Para finalizar os aspectos introdutórios, vale ressaltar, ainda, as atividades de disjunção que se operaram sobre o Conhecimento (considerado aqui em sua forma totalizante), para organizá-lo, em uma primeira instância, em tipos distintos (popular, filosófico, religioso, científico, etc.). Além dessa primeira disjunção, "a complexidade do universo e a multiplicidade de fenômenos que nele se manifestam, aliadas à necessidade do ser humano de estudá-los para poder entendê-los e explicá-los, levaram ao surgimento de diversos ramos de estudo e ciências específicas", organizados "quer de acordo com sua ordem de complexidade, quer de acordo com seu conteúdo: objeto ou temas, diferença de enunciados e metodologia empregada"(9:22).

A divisão do Conhecimento em conhecimentos, áreas de estudo e ciências específicos efetuou a organização de diferentes campos (ou domínios) do saber; efetuou a construção de casas específicas de conhecimento, sob cujos tetos se abrigam as diferentes disciplinas que produzem conhecimento, seja teórico ou prático, e as "confrarias" dos cientistas a elas ligados. Entre essas diferentes casas são, às vezes, construídos muros simbólicos, alguns dos quais praticamente intransponíveis para aqueles que nelas usualmente não se abrigam, que não sejam "parentes" ou considerados, no mínimo, "vizinhos desejáveis".

A cada casa de conhecimento corresponde uma subcomunidade científica relativamente bem definida (a "confraria" dos cientistas a ela ligados), cujos membros se reconhecem entre si e compartilham uma cultura própria em termos de objeto, linguagem e arsenal metodológico e técnico, os quais configuram um modo particular de conhecer, que se pretende seja reconhecido, também, externamente ${ }^{(10)}$. Cada subcomunidade científica aponta quais são os padrões, formas e estruturas particulares que lhe servem de guia em termos de construção de conhecimento e que exemplificam maneiras ou abordagens características de pensar sobre os fenômenos que estão em seu domínio de interesse.

O saber resultante da atividade de conhecer e de produzir conhecimento de cada subcomunidade científica particular se materializa com a explicitação dos conceitos representativos dos fenômenos que indicam e delimitam seu domínio de interesse; da(s) filosofia(s) que expressa(m) suas crenças e valores em relação aos fenômenos que estão em seu domínio de interesse; enfim, de teorias que articulam, em proposições testáveis no mundo empírico, os conceitos representativos dos fenômenos que estão em seu domínio de interesse. Com a construção da casa do conhecimento da Enfermagem, não foi ou está sendo diferente.

\section{As teorias de enfermagem e a construção do conhecimento} da área

Como resultado da atividade de atribuir significado ao mundo da Enfermagem, as enfermeiras têm, ao longo do tempo, não só elaborado, como comunicado, de modo sistemático, um corpo de conhecimento específico, aplicável em qualquer dos campos da prática profissional - ensino, pesquisa ou assistência. Esse corpo de conhecimento não é estático; ao contrário, ele vem se transformando, assumindo modos de expressão diversos em sua trajetória de construção(11). Um desses modos está representado pelas teorias de enfermagem, expressão formal do padrão empírico de conhecimento, ou da Ciência da Enfermagem, conforme a classificação proposta por Barbara Carper em 1978. ${ }^{(12)}$ Para construir esse conhecimento, as enfermeiras têm identificado e definido conceitos representativos de fenômenos que estão em seu campo de interesse, e inter-relacionado esses conceitos em proposições teóricas que refletem visões específicas acerca desses fenômenos e que determinam, potencialmente, inovações, evoluções e/ou revoluções no saber e no fazer da Enfermagem.

Falar sobre a contribuição das teorias de enfermagem para a construção do conhecimento da área nos remete, quase que obrigatoriamente, a Florence Nightingale, nosso mito de origem, cujas ações e publicações Ihe renderam o reconhecimento como fundadora da Enfermagem moderna. A rigor, não se pode afirmar que Florence, em seus escritos, tenha elaborado uma teoria de enfermagem, mas uma filosofia para a prática de enfermagem, que serviu de base a boa parte dos modelos conceituais ou das teorias de enfermagem contemporâneas. Em Notes on nursing, seu livro mais amplamente conhecido, ela reúne os conhecimentos que havia adquirido no trato com doentes, enfermeiras e hospitais em um certo número de princípios, que deveriam servir de fundamento para as mulheres que, "em algum período de sua vida", assumissem "a responsabilidade pessoal pela saúde de alguém" (13:3). Esses princípios se relacionam aos seguintes fenômenos: ventilação (ou ar fresco), iluminação, aquecimento, quietude (ou silêncio), limpeza, pontualidade e cuidado na administração da alimentação. A falta de conhecimento ou de atenção a esses fenômenos retardaria "o processo restaurador que a Natureza instituiu e a que nós chamamos doença"(13:8). Em suas palavras, o que se "deve fazer (...) é colocar o paciente em condições tais que a Natureza possa agir sobre ele"(14:77). Portanto, atuando sobre esses fenômenos, estar-se-ia favorecendo o sucesso dos processos restauradores da Natureza e, conseqüentemente, favorecendo a manutenção da capacidade vital do paciente.

Embora as idéias de Florence Nightingale tenham sido divulgadas na segunda metade do século XIX, foi somente a partir da década de 1950, que se começou a articular e sistematizar novas visões teórico-filosóficas acerca da Enfermagem. Esse fato coincide com a inserção, na década de 1950, nos Estados Unidos, da Enfermagem entre as carreiras de nível superior ${ }^{(15,16)}$. Em virtude da exigência, na carreira universitária, de realização de cursos de pós-graduação, cresce naquele país, a partir de então, o número de profissionais com título de mestrado e doutorado.

Ao serem introduzidas, durante os cursos de pósgraduação, no mundo da Filosofia da Ciência, as enfermeiras passaram a refletir, de modo sistemático e deliberado, acerca 
da natureza da Enfermagem e dos propósitos para os quais a profissão existia. Em conseqüência, passou-se a proclamar a urgência da constituição de um corpo de conhecimento substantivo que pudesse ser identificado como a Ciência da Enfermagem e que, sobretudo, viabilizasse o reconhecimento externo da profissão como um ramo específico de estudo e de produção de conhecimento científico ${ }^{(16)}$. Desenvolver tal corpo de conhecimento passou a ser visto como uma questão de vital importância para a sobrevivência e evolução da Enfermagem pois, a profissão não poderia "continuar a existir sem que tornasse explícitas suas bases teóricas para a prática, de forma que esse conhecimento pudesse ser comunicado, testado e expandido"(17:204).

Voltado, pois, para o preenchimento da lacuna existente no que diz respeito à necessidade de descrever, explicar, predizer ou controlar os fenômenos que pertencessem ao seu domínio de interesse a partir de referenciais próprios, esse novo enfoque forneceu o ímpeto para o desenvolvimento de teorias de enfermagem, assim como para o estabelecimento de dispositivos que assegurassem a utilização desse conhecimento na prática.

Conforme é sabido, o marco inicial para o desenvolvimento de referenciais teóricos próprios da área foi a publicação, em 1952, do livro de Hildegard Peplau abordando o relacionamento interpessoal em enfermagem. A partir de então, outras teóricas norte-americanas desenvolvem e publicam novas teorias de enfermagem, em que selecionam e inter-relacionam, a partir de diferentes pontos de vista filosóficos, conceitos que refletem a natureza e o escopo da Enfermagem.

De acordo com Meleis ${ }^{(18)}$, a análise das teorias de enfermagem permite que sejam classificadas de dois modos distintos. No primeiro, a classificação é feita a partir da identificação do foco primário, o que possibilita distinguir quatro grupos de teorias de enfermagem: 1) as centradas no cliente; 2) as centradas no relacionamento entre o cliente e o meio ambiente; 3) as centradas nas interações enfermeira-cliente; e 4) as centrados na terapêutica de enfermagem. No segundo modo, a classificação das teorias é feita tendo por base o papel que as enfermeiras desempenham na prática profissional e a escola de pensamento a que as teóricas que as elaboraram estavam ou estão ligadas. Nesse modo, distinguem-se três grupos de teorias de enfermagem: 1) as orientadas para as necessidades dos clientes (o "que" do cuidado de enfermagem), elaboradas por teóricas identificadas com a escola de pensamento do Teacher's College da Universidade de Columbia, como Faye Abdellah, Virginia Henderson e Dorothea E. Orem; 2) as orientadas para o processo de interação enfermeira-cliente (o "como" do cuidado de enfermagem), elaboradas por teóricas identificadas com a escola de pensamento da Escola de Enfermagem da Universidade de Yale, como Imogene M. King, Ida Jean Orlando, Josephine Paterson e Loretta Zderad, Hildegard Peplau, Joyce Travelbee e Ernestine Wiedenbach; e 3) as orientadas para os resultados das ações de enfermagem (o "porque" do cuidado de enfermagem), elaboradas por teóricas identificadas com a escola de pensamento conhecida como Leste/Oeste (ou Nova Iorque/Los Angeles) e que tem como representantes principais Dorothy Johnson e Martha Rogers, além de Myra Estrin Levine e Sister Callista Roy.

Como foi afirmado inicialmente, o conhecimento e o conhecer são fenômenos evolutivos, sujeitos a enriquecimentos e a alterações progressivos, que se destinam às nossas necessidades de ajuste intelectual com o meio ambiente, em um dado espaço e tempo. Assim, a partir do final da década de 1970, observa-se ter ocorrido uma importante mudança na orientação paradigmática das teorias elaboradas no âmbito da Enfermagem as quais, ao invés de refletir a perspectiva funcionalista predominante nas décadas anteriores, passam a incorporar dimensões qualitativas que caracterizam o papel social da profissão, não a partir do que as enfermeiras fazem, mas a partir do que se pode afirmar que seja a essência da Enfermagem ${ }^{(16)}$. Nesse contexto, várias teóricas, como Madeleine Leininger, Jean Watson, Patricia Benner e Judith Wrubel, Delores Gaut, Peggy Chinn, entre outras, baseadas em concepções filosóficas humanistas, elaboraram ou estão elaborando teorias do cuidado, fenômeno esse visto por essas teóricas como o cerne, o coração ou a própria alma da Enfermagem. Essas teorias cultivam o reconhecimento tácito da significância dos padrões estético, ético e pessoal de conhecimento para a Enfermagem, os outros três padrões fundamentais de conhecimento, além do empírico, que foram descritos por Barbara Carper em 1978(16).

A divulgação e subseqüente utilização na prática profissional (ensino, pesquisa e assistência) desses referenciais teóricos não se limitou, como é de se supor, à cultura de enfermagem norte-americana, extrapolando suas fronteiras para o âmbito internacional e, inclusive, estimulando elaborações semelhantes por enfermeiras de outros países.

No Brasil, a primeira enfermeira a falar sobre teoria no campo profissional foi Wanda de Aguiar Horta, que procurou despertar a Enfermagem brasileira para a importância do assunto $^{(11)}$. Sua obra reflete inicialmente o empenho na divulgação do conhecimento acerca de teorias elaboradas pelas enfermeiras norte-americanas e do processo de enfermagem, entendendo-o como o instrumento metodológico por meio do qual esses referenciais seriam aplicados na prática profissional. Posteriormente, faz ela própria o esforço de síntese teórica e divulga, em 1974, a teoria das necessidades humanas básicas.

A partir do pioneirismo da Dra. Wanda de Aguiar Horta, o conhecimento a respeito das teorias de enfermagem tem avançado consideravelmente no meio da enfermagem brasileira ${ }^{(11)}$, para o que têm contribuído os cursos de pósgraduação stricto sensu da área e a constituição de grupos de estudo e pesquisa sobre a fundamentação, tecnologia e instrumentação da Enfermagem. Em alguns desses cursos ou grupos, além de se promover a discussão sobre as teorias de enfermagem já existentes, ou sobre teorias elaboradas em outros campos do conhecimento, tem-se estimulado a reflexão teórico-filosófica, seja a de caráter pessoal/individual ou a grupal/coletiva, e essas atividades têm resultado em alguns produtos concretos, tais como a teoria sistêmico-ecológica, que vem sendo desenvolvida por Rosalda Paim(19); o modelo de organização da enfermagem, proposto por Liliana F. Daniel(20); o referencial do cuidado holístico-ecológico, elaborado por Zuleica Maria Patrício(21); ou a teoria de intervenção práxica da enfermagem em saúde coletiva, descrita por Emiko Yoshikawa Egry ${ }^{(22)}$, entre outros exemplos possíveis.

Além disso, concorrendo para a expansão do arsenal metodológico utilizado pela área no processo de conhecer, vale destacar a modalidade de pesquisa convergente-assistencial proposta por Mercedes Trentini e Lygia Paim, que representa para as profissionais de enfermagem, nas palavras das autoras, "um instrumento útil para 'aprender a pensar o fazer' no seu cotidiano, pesquisando as implicações teóricas e práticas do seu fazer"(23:28). Partindo do pressuposto de que o ambiente concreto da prática profissional é o lugar por excelência para se testar a validade do conhecimento que tem sido gerado no âmbito da Enfermagem, pode-se afirmar que a pesquisa convergenteassistencial é uma modalidade bastante adequada ao teste das teorias de enfermagem disponíveis na literatura da área.

Pelo exposto, não nos restam dúvidas de que as teorias desenvolvidas no âmbito da Enfermagem têm concorrido para explicitar a complexidade e multiplicidade de conceitos representativos dos fenômenos que definem e delimitam seu campo de interesse, assim como para explicitar as múltiplas perspectivas a partir das quais é possível expressar as crenças e valores da área em relação a esses fenômenos. Em seu processo evolutivo, as teorias de enfermagem têm, pois, 
contribuído para a formação de uma base relativamente sólida de conhecimento, que organiza o mundo fenomenal da Enfermagem em torno de quatro conceitos centrais, ou domínios temáticos, amplamente reconhecidos como os mais recorrentes na literatura teórica da área - enfermagem, ser humano, meio ambiente (físico, social e simbólico) e saúde -, considerados como a matriz disciplinar da Enfermagem.

Os quatro conceitos centrais, assim como outros conceitos que a eles se articulam nas proposições teóricas elaboradas pelas autoras das teorias da área, participam da construção da linguagem especial da Enfermagem, ou seja, da construção do vocabulário particular utilizado pelo grupo sócio-profissional constituído pelos profissionais e ocupacionais de enfermagem, em face do interesse comum, ou do fim comum de nomear os fenômenos ou as atividades pertinentes à profissão.

A linguagem é parte da vida social dos grupos humanos ${ }^{(24)}$. Como tal, é sujeita a acréscimos vocabulares e a alterações dinâmicas no significado discursivo e/ou denotativo de termos já incorporados a seu léxico. Do mesmo modo que ocorre com o linguajar de uma determinada formação sóciocultural, a linguagem técnica de um campo específico de conhecimento, em sua evolução histórica, está sujeita a novidades e a modismos vocabulares ${ }^{(25)}$. Isso também ocorre na linguagem especial da Enfermagem, em que podem ser identificados, além de acréscimos vocabulares, modismos terminológicos correspondentes a etapas específicas, por exemplo, da história das políticas públicas e práticas sociais da área da saúde, do desenvolvimento técnico-científico da área da saúde ou da própria profissão. Em determinados momentos dessa história alguns termos inflacionam verdadeiramente nosso vocabulário técnico, mesmo que, sobre alguns deles, paire uma verdadeira cortina de fumaça semântica obscurecendo seu significado e dificultando a compreensão e o reconhecimento intersubjetivo dos fenômenos a que se referem.

Vale ressaltar que garantir a compreensão e o reconhecimento intersubjetivo do significado dos conceitos incluídos na linguagem especial da Enfermagem é, a nosso ver, um aspecto básico, pois eleva seu potencial de aplicabilidade prática, seja no ensino, na pesquisa ou na assistência de enfermagem. Se temos a pretensão de promover a Ciência da Enfermagem, precisamos tornar objetiva a linguagem que a expressa; precisamos situar os termos que a constituem em um universo comum de percepção e de comunicação - o universo instituído pela cultura da Enfermagem. É preciso ter a clareza de que atribuir um nome (rótulo lingüístico) a fenômenos específicos representa apenas o comeco da caminhada pois, ao nomear uma entidade prélingüística, nós estamos admitindo formalmente sua existência e criando a possibilidade para que se construa e se promova, de modo sistemático e contínuo, o conhecimento acerca do significado desse fenômeno(26).

Tomemos como exemplo, entre tantos outros possíveis, o fenômeno cuidado de enfermagem. Talvez porque esse fenômeno fosse algo tão óbvio, tão internalizado em nosso mapa conceitual, durante um bom período de tempo nos esquivamos de examinar, avaliar e promover seu significado no âmbito da prática profissional. Atualmente, ao contrário, além das teorias que o abordam como tema central, há disponível uma vasta literatura em que se abordam diferentes fenômenos subjacentes ao cuidado de enfermagem, tais como conforto, toque, apoio, esperança, reciprocidade, espiritualidade, confiança, etc.; em que se apontam que aspectos constituintes da conduta humana são necessários para que o cuidado se manifeste; em que se descrevem como o cuidado é experimentado pelos pacientes e pelas enfermeiras ${ }^{(27)}$; e em que, utilizando-se da riqueza vocabular disponível, se complementa o termo cuidado com uma variedade de adjetivos que o carregam de dimensões semânticas às vezes distintas, outras vezes semelhantes ou associadas. Essa reflexão teórica tem resultado em uma ampliação de nosso entendimento acerca do fenômeno cuidado de enfermagem e tem gerado inovações, evoluções e/ou revoluções metodológicas e tecnológicas na prática profissional.

Ressalte-se haver, ainda, na atividade de reflexão teóricofilosófica que focaliza conceitos relacionados a fenômenos particulares de interesse para a área, a possibilidade de construção de teorias de enfermagem de amplitude média, mais aderentes à prática profissional porque, via de regra, são desenvolvidas pari passu com a execução de pesquisas diretamente vinculadas a questões ou problemas que emergem da própria prática profissional. Há, na linguagem especial da Enfermagem, um número considerável de conceitos que estão reclamando por esse tipo de empreendimento.

\section{Considerações finais}

Pode-se afirmar que as teorias de enfermagem colocam em nossas mãos a possibilidade de reflexão criativa e o domínio do nosso processo de trabalho, além de uma possível ruptura com a tradicional execução de tarefas complementares ao ato médico ${ }^{(28)}$. Entretanto, fazer a seleção da teoria de enfermagem que vai guiar a prática profissional nem sempre é uma tarefa fácil pois, conforme foi afirmado, cada uma das teorias disponíveis está organizada a partir de diferentes visões de mundo e descrevem e inter-relacionam de modo particular os quatro conceitos centrais da disciplina - ser humano, meio ambiente, saúde e enfermagem ${ }^{(29)}$. Essa seleção requer, pois, um conhecimento suficientemente aprofundado das diversas teorias disponíveis e das variáveis que definem a situação específica do cliente ou do grupo de clientes de quem se vai cuidar.

Para finalizar, lembramos que a Enfermagem tem sido proclamada por seus exercentes como uma ciência e uma arte. Da mesma forma que, em uma galeria de arte, não é o conhecimento do artista ou o instrumental que utilizou o que importa, mas a obra artística criada por ele ${ }^{(30)}$, pode-se afirmar que os referenciais teóricos ou os instrumentos metodológicos e tecnológicos próprios da área não são a Enfermagem. Enfermagem é o que se cria usando esse conhecimento e esses instrumentos.

\section{Referências}

1. Buzzi AB. Introdução ao pensar. $11^{\mathrm{a}}$ ed. Petrópolis (RJ): Vozes; 1983.230p.

2. Hegenberg L. Explicações científicas: introdução à filosofia da ciência $2^{\mathrm{a}}$ ed. São Paulo: Epu/Edusp;1973.310p.

3. Hegenberg L. Significado e conhecimento. São Paulo: Epu/ Edusp;1975.185p.

4. Ferreira $\mathrm{ABH}$. Novo dicionário da língua portuguesa. $2^{\mathrm{a}}$ ed. Rio de Janeiro:Nova Fronteira;1986. Conhecimento;1809p. p.454

5. Morin E. O método 3: o conhecimento do conhecimento. Porto Alegre (RS): Sulina;1999.309p.

6. Kim HS. Terminology in structuring and developing nursing knowledge In: King IM, Fawcett J, editors. The language of nursing theory and metatheory. Indianapolis (IN): Sigma Theta lau International;1997. 98p.p.27-36.

7. Castim F. Teoria da linguagem - tópicos. 9a ed. rev. ampl. Recife (PE): Fundação Antônio dos Santos Abranches - Fasa;1994.74p.

8. Koch IV. A inter-ação pela linguagem. São Paulo: Contexto;1992.115p. (Coleção Repensando a língua portuguesa)

9. Lakatos EM, Marconi MA. Metodologia científica. $2^{a}$ ed. São Paulo: Atlas; 1992.249p.

10. Fourez G. A construção das ciências: introdução à filosofia e à ética das ciências. São Paulo: Editora da Unesp;1995.319p.

11. Souza MF. Modelos teóricos e teorias de enfermagem: contribuição para a construção do conhecimento em enfermagem no Brasil. In: Garcia TR, Pagliuca LMF. A construção do conhecimento em enfermagem: coletânea de trabalhos. Fortaleza (CE): RENE;1998. 169p.p.51-63. 
12. Carper BA. Fundamental patterns of knowing in nursing. In: Nicoll LE, editors. Perspectives on nursing theory. $2^{\text {nd }}$ ed. Philadelphia (PA): Lippincott;1992.696p.p.216-24.

13. Nightingale F. Notes on nursing: what it is and what it is not. New York: Dover Publications;1969.140p.

14. Baly M. As Miss Nightingale said... $2^{\text {nd }}$ ed. London: Baillière Tindall; 1997.138p.

15. Chinn PL, Kramer MK. Theory and nursing: a systematic approach. $4^{\text {th }}$ ed. St. Louis (MO): Mosby;1995.235p.

16. Chinn PL, Kramer MK. Theory and nursing: integrated knowledge development. $5^{\text {th }}$ ed. St. Louis (MO): Mosby;1999.265p.

17. Johnson DE. The nature of a science of nursing. In: Nicoll LE, editor. Perspectives on nursing theory. $2^{\text {nd }}$ ed. Philadelphia (PA): Lippincott;1992.696p.p.189-95.

18. Meleis Al. Nursing theory: an elusive mirage or a mirror of reality. In: Meleis Al. Theoretical nursing: development and progress. $2^{\mathrm{a}} \mathrm{ed}$. Philadelphia (PA): Lippincott; 1991.617p.p.249-67.

19. Paim R. Teoria sistêmico-ecológica: uma visão holística da enfermagem. Lambari (MG): CEL - Informática \& Editoração;1998. $134 p$.

20. Daniel LF. Enfermagem: modelos e processos de trabalho. São Paulo: Epu;1987.117p.

21. Patrício ZM. Ser saudável na felicidade-prazer: uma abordagem ética e estética pelo cuidado holístico-ecológico. Pelotas (RS): Editora Universitária/UFPel; Florianópolis (SC): PPG em Enfermagem/UFSC; 1996.153p.

22. Egry EY. Saúde coletiva: construindo um novo método em enfermagem São Paulo: Ícone;1996.114p.

23. Trentini M, Paim L. Pesquisa em enfermagem: uma modalidade convergente-assistencial. Florianópolis (SC): Editora da UFSC;1999. $162 p$.
24. Hymes D. [Posfácio]. In: Burke P, Porter R, organizadores. Linguagem, indivíduo e sociedade: história social da linguagem. $1^{a}$ reimp. São Paulo: Editora da Universidade Estadual Paulista;1993.465p.p.431-53.

25. Porter R. "Expressando sua enfermidade": a linguagem da doença na Inglaterra georgiana. In: Burke $\mathrm{P}$, Porter R, organizadores. Linguagem, indivíduo e sociedade: história social da linguagem. $1^{\mathrm{a}}$ reimp. São Paulo: Editora da Universidade Estadual Paulista; 1993. 465p.p.365-94.

26. Knafl KA. Concept development. In: NANDA. Monograph of the invitational conference on research methods for validating nursing diagnosis. Palm Springs (CA): NANDA;1989.332p.p.37-63.

27. Brykczynska G. A brief overview of the epistemology of caring. In: Brykczynska G, editor. Caring: the compassion and wisdom of nursing. San Diego (CA):Singular Publishing Group;1997.209p.p.19.

28. Pires D. Construir um novo saber de enfermagem: um dos nossos desafios para a virada do século. In: CBEn. Anais do $42^{\circ}$ Congresso Brasileiro de Enfermagem, Construção de uma nova prática;1990 out 11-16; Natal (RN), Brasil. Natal (RN):ABEn; 1990.144p.p.69-75.

29. Kenney JW. Relevance of theoretical approaches in nursing practice. In: Christensen PJ, Kenney JW. Nursing process: application of conceptual models. $3^{\text {rd }}$ ed. St. Louis (MO): Mosby;1990. 367p.p.319.

30. Rose P. Science and technology: tools in the creation of nursing. In: Marks-Maran D, Rose P, editors. Reconstructing nursing: beyond art and science. London: Baillière Tindall;1997.192p.p.26-52.

Data de Recebimento: 22/01/2004

Data de Aprovação: 28/06/2004 